\title{
CRONOLOGía GLACIAR PLEISTOCENA DE LA SERRA DE GERÊS, (NORTE DE PORTUGAL)
}

\author{
J.R. VIDAL ROMANI* \& D. FERNÁNDEZ MOSQUERA
}

\begin{abstract}
Pleistocene glacier chronology of the Serra do Gerês (Northern Portugal) — The dating of glacial features by cosmogenic ${ }^{21} \mathrm{Ne}$ isotopes in the Serra do Gerês have permitted to distinguish several glacier phases in which until the moment was considered a single one attributed to the Würm. The resulting data are roughly consistent with the previous geomorphologic model for the area and improves the chronological accuracy and the understanding of the pleistocene glacial dynamics in Northern Portugal.

Keywords: Pleistocene, cosmogenic dates, Serra do Gerês, Portugal, Spain, Glaciarism.

Resumo

Cronologia glaciaria plistocénica da Serra do Gerês - A datagem de superfícies rochosas com evidências de glaciação na Serra do Gerês, através de isótopos cosmogónicos de ' $\mathrm{Ne}$ permitiu distinguir várias fases glaciarias numa área onde, até ao momento, apenas se considerava uma única, atribuída ao Würm. Os dados obtidos são no geral consistentes com o modelo geomorfológico existente, e melhoram o conhecimento da cronologia dos episódios glaciarios e da dinâmica glaciaria plistocénica do Norte de Portugal.
\end{abstract}

Palavras-chave: Plistocénico, datagens, Serra do Gerês, Portugal, Espanha, Glaciarismo.

\section{ANTECEDENTES}

Los primeros trabajos sobre el glaciarismo pleistoceno del NW de la Península Ibérica tienen ya mas de un siglo (CHOFFAT, 1894), aunque se trate de meras descripciones morfológicas. No es hasta fechas mas recientes, (SCHMIDT-THOMÉ, 1973, 1978, 1983; COUDÉ-GAUSSEN, 1981; VIDAL ROMANI et al., 1990a y b; BRUM et al, 1992), cuando comienza a estudiarse de manera sistemática el glaciarismo de Serra do Gerês. Los depósitos glaciares mas frecuentes en la zona son los till s.l y las morrenas frontales o laterales, (SCHMIDT-THOME, 1973, 1978, 1983; COUDÉGAUSSEN, 1981; VIDAL ROMANI et al, 1990a y b; BRUM et al, 1992). Sin embargo el problema principal es que los till, aun en cortes frescos, se confunden, por personas no expe-rimentadas, con suelos o con el mismo regolito. Las morrenas formadas esencialmente por bolos residuales son lo mas aparente en superficie, aunque también han sido confundidas habitualmente con depósitos residuales o de vertiente, (VIDAL ROMANI et al, 1990a y b; BRUM et al, 1992). Por esta razón algunos autores han puesto en duda la existencia de glaciarismo pleistoceno en el NW portugués, (TEIXEIRA \& CARDOSO, 1979; MARTINEZ DE PISÓN \& ARENILLAS PARRA, 1979, 1984), aunque la mayoría de investigadores lo hayan apoyado claramente, (COUDÉ-GAUSSEN, 1981; SOARES DE CARVALHO \& LOPES NUNES, $1981 \mathrm{a}$ y b; VIDAL ROMANí et al, 1990a y b). En ninguno de los trabajos antes citados se pudieron hacer dataciones absolutas de los sedimentos, aunque ciertos autores admitían implícitamente que el glaciarismo descrito correspondía al
Würm, considerado además como el período mas frío y de máximo avance glaciar en el NW ibérico. Los únicos datos con excepción de los geomorfológicos, que, de alguna manera, permiten reconstruir la parte final de la época glaciar en la Serra do Gerés son los proporcionados por los estudios de turberas de las zonas altas de la Sierra. A partir de ellos ha sido posible establecer la evolución climática y de la cubierta vegetal de la zona principalmente durante el postglaciar y en casos excepcionales desde un poco antes de su final. Es ahí donde se han podido realizar las únicas, hasta el momento, dataciones absolutas para el Cuaternario en la zona del trabajo. Las primeras investigaciones palinológicas (BELLOT RODRÍGUEZ, 1952), son para diferenciar las especies de Pinus presentes en la Sierra. Del análisis polínico de once turberas, sin dataciones absolutas, se identifica Pinus sylvestris como especie relicta en la Sierra mientras que se estima que Pinus pinaster es de implantación reciente. En otro contexto se halla el trabajo de COUDÉ-GAUSSEN \& DENÉFLE (1980) y COUDÉGAUSSEN (1981) que analizan las turberas de Lagoa de Marinho (1170 $\mathrm{m}$ de altitud; $140 \mathrm{~cm}$ de profundidad) y Lamas de Vez $(1180 \mathrm{~m}$ de altitud; $200 \mathrm{~cm}$ de profundidad), situadas en la zona glaciada durante el Pleistoceno. La datación de la base de Lamas de Vez por ${ }^{14} \mathrm{C}$ es muy reciente, entre $1.170 \pm 80$ B.P. y $990 \pm 60$ B.P. El paisaje vegetal reflejado en ambas secuencias es un paisaje abierto, antropizado, dominado por ericáceas fundamentalmente. Dos equipos de investigadores dirigidos por RAMIL REGÓ \& Ruiz ZAPATA vuelven a estudiar la Lagoa de Marinho una pequeña cubeta yuxtaglaciar limitada por un cordón 
morrénico lateral correspondiente al máximo avance del Glaciar de Couce. En ella, RAMIL REGÓ et al. (1993) datan por ${ }^{14} \mathrm{C}$ la secuencia: entre $280-290 \mathrm{~cm}$ $(10.910 \pm 90$ B.R) y entre $40-50 \mathrm{~cm}(7.500 \pm 70$ B.R). El análisis polínico representa un paisaje tardiglaciar dominado por Pinus con temprana expansión de Quercus lo que correspondería con un clima benigno. El descenso de táxones arbóreos con aumento de herbáceas (Poaceae y Artemisia) corresponde según estos autores al Dryas Reciente, caracterizándose el Holoceno por la fuerte expansión de Quercus, con un óptimo a $7.500 \pm 70 \mathrm{BP}$, y la aparición de polen de cereal, entendido como el inicio de la antropización, hacia $5 \mathrm{ka}$. BP. La evolución más reciente del paisaje está representada por la desaparición del arbolado y el dominio de las formaciones arbustivas (Ericaceae, Calluna) como ya habían señalado mucho antes COUDÉ-GAUSSEN \& DENÉfLE (1980). Simultáneamente Ruiz ZAPATA et al. (1993) publican otro estudio de la Lagoa de Marinho con un sondeo de $210 \mathrm{~cm}$ en el que obtienen dataciones diferentes, 8.450 BP, 7.400 BP y $2.140 \mathrm{BP})$, y con un espectro polínico distinto al definido por RAMIL REGÓ et al. (1993). Los datos polínicos de Ruiz ZAPATA et al. (1993) sugieren bosques densos de Pinus hasta la fecha de 2.140 BP para las cotas más altas y Betula y Quercus para las más bajas. El paisaje reciente estaría dominado por Ericaceae y nueva expansión de Pinus junto con Castanea, Olea y Poaceae. Posteriormente RAMIL REGÓ et al. (1996) comparan sus resultados de la Laguna de Marinho con los de Ruiz ZAPATA et al. (1993) achacando las diferencias existentes entre ambos diagramas polínicos a un hiato sedimentario. En un trabajo posterior, RAMIL REGÓ et al. (1998)

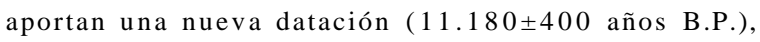
sin especificar ni lugar ni profundidad de la muestra. Los autores extrapolan una edad para la base del sondeo hasta $14 \mathrm{ka}$ años BP, que así cubriría, según ellos, del Dryas Antiguo a la actualidad. Tantas diferencias, en la investigación de la Lagoa de Marinho, solo se explican si ambos equipos han estudiado distintas partes de una misma secuencia aunque el sondeo podría ser aun mas antiguo pues la edad de las morrenas que delimitan la Lagoa de Marinho puede superar los $300 \mathrm{ka}$ años (VIDAL ROMANI et al, 1999).

Sin embargo los datos geomorfológicos, (VIDAL ROMANÍ et al., 1990a y b), hacían presumir un mayor lapso de tiempo entre el máximo glaciar, correspondiente a los frentes morrénicos mas avanzados, y el fin de los glaciares de Gerés. Inicialmente se había asumido basándose en las similaridades geomorfológicas, una cronología y modelo de deglaciación únicos para todo el borde norte peninsular desde el Pirineo catalán, (Bordonau; 1992), y otras zonas de la Cadena Cantábrica, (JIMÉNEZ SÁNCHEZ, 1996; LEIRA \& VIDAL ROMANI, 1997), hasta Galicia y Norte de Portugal. Este esquema, aceptado por algunos autores, (BORDONAU, 1992; BRUM FERREIRA et al, 1992;
JIMÉNEZ SÁNCHEZ, 1996; LEIRA \& VIDAL ROMANI, 1997; SOARES DE CARVALHO \& LOPES NUNES, 1981a y b; VILAPLANA, 1981), tenía el inconveniente de no contar con una base de dataciones absolutas (las que existían raramente superaban los $10 \mathrm{ka}-15 \mathrm{ka}$ antes de ahora). Con estos antecedentes es evidente que solo los datos geomorfológicos permitían conocer con un grado de detalle apreciable el desarrollo del glaciarismo pleistoceno en el área aunque sin ninguna referencia cronológica absoluta. Sin embargo una técnica de aplicación reciente: la datación por isótopos cosmogónicos en cristales de cuarzo, ha venido a abrir una nueva via para el estudio de la cronología del glaciarismo en zonas como la Serra de Gerés Como ya se dijo anteriormente, en ésta área el substrato es granítico (granodiorita). Las zonas sometidas a la glaciación pleistocena debían estar ya fuertemente alteradas, (COUDÉ-GAUSSEN, 1981) antes del Pleistoceno. La erosión glaciar, (VIDAL ROMANI et al, 1990a y b), contribuyó, primero a eliminar el regolito, erosionando después la roca al menos durante las etapas glaciares. En Gerés las superficies rocosas glaciadas conservan aun restos de superficies de pulido, (SOARES DE CARVALHO \& LOPES NUNES, 1981a y b; VIDAL ROMANI et al, 1990a y b). En las zonas donde la desaparición del hielo glaciar fue mas tardía (Circo de Cocoes do Concelinho), la degradación postglaciar no fue demasiado severa (SOARES DE CARVALHO \& LOPES NUNES, 1981a y b), se pudo evaluar la erosión postglaciar en no mas de 15 milímetros para los últimos $15 \mathrm{ka}$ años, lo que es un valor insignificante para este trabajo. Puede considerarse que las superficies rocosas glaciadas durante el Pleistoceno en la Serra de Geréz están prácticamente intactas, lo que permite utilizar la técnica de datación (CERLING \& CRAIG, 1994), por isótopos cosmogónicos generados en los cristales de cuarzo por la interacción de los rayos cósmicos con las superficies graníticas expuestas por la erosión en nuestro caso glaciar.

\section{MATERIAL Y MÉTODOS}

\subsection{Situación geomorfológica de las muestras}

La Serra do Gérez-Xurés se sitúa entre el Norte de Portugal y el Sur de la provincia de Ourense, (Galicia, España). En su mayor parte incluida en el Parque Nacional portugués da Peneda-Géres el acceso y toma de muestras, excepto en la parte española, están prohibidos por las normas legales. Por esta razón las muestras fueron recogidas en la vertiente gallega (ver figura 1) de la Serra de Gerés. Según diversos autores (BRUM et al, 1992, 1999; VIDAL et. al, 1990 a y b), la glaciación de la Serra de Gerés solo afectó a la zona española en los momentos de máximo glaciar ya que la alimentación de las lenguas que dieron lugar 
a las morrenas frontales de Fecha, Curro y Vilamés (VIDAL et al, 1990a y b), se produce no por la generación de glaciares en la zona española sino por difluencia del hielo desde la zona portuguesa a la española. La primera muestra (X-1), analizada corresponde a una superficie de pulido glaciar situada en la Pórtela da Amoreira (ver figuras 1 y 2), en el fondo de un pequeño valle de la cabecera del río Vilamés y por encima de la Mina das Sombras. La muestra, X-2 delimitada por 3 cordones morrénicos sucesivos está en la vertiente oeste del valle del Río Vilamés y corresponde a una superficie de pulido glaciar (ver figura 1). Según los trabajos previos (VIDAL et al, 1990a y b; B RUM et al, 1992), correspondería a la etapa de máximo glaciar en la vertiente española, y obviamente en toda el área.

\subsection{Localización geográfica de las muestras}

Todas las muestras fueron situadas, en posición y altitud, mediante GPS (Global Position System). Asimismo fueron situadas (ver figura 1 y tabla 1), con respecto a los frentes morrénicos y superficies de pulido mas relevantes.

\subsection{Toma de muestras}

La toma de muestras se realizó con un taladro portátil para roca ROY Modelo D026-GT10 de $50 \mathrm{~cm} \mathrm{de}$ largo equipado con una broca de corona de diamante de 2 pulgadas de diámetro. Los testigos alcanzaron una longitud máxima de $0,5 \mathrm{~m}$.

\subsection{Preparación de las muestras}

En primer lugar la superficie de los testigos fue limpiada mecánicamente. Se cortaron los 5 primeros $\mathrm{cm}$ de cada testigo en pequeños trozos para molerlos en una trituradora de mandíbula y separarlos en 4 fracciones según su tamaño de grano: $0>2 \mathrm{~mm}$; 0 O, $95 \mathrm{~mm}<0<2 \mathrm{~mm} ; \quad$ O $, 5 \mathrm{~mm}<0<0,95 \mathrm{~mm} ; 0<0,5 \mathrm{~mm}$. Todas las fracciones fueron seleccionadas a la lupa para escoger la fracción más limpia. Una vez

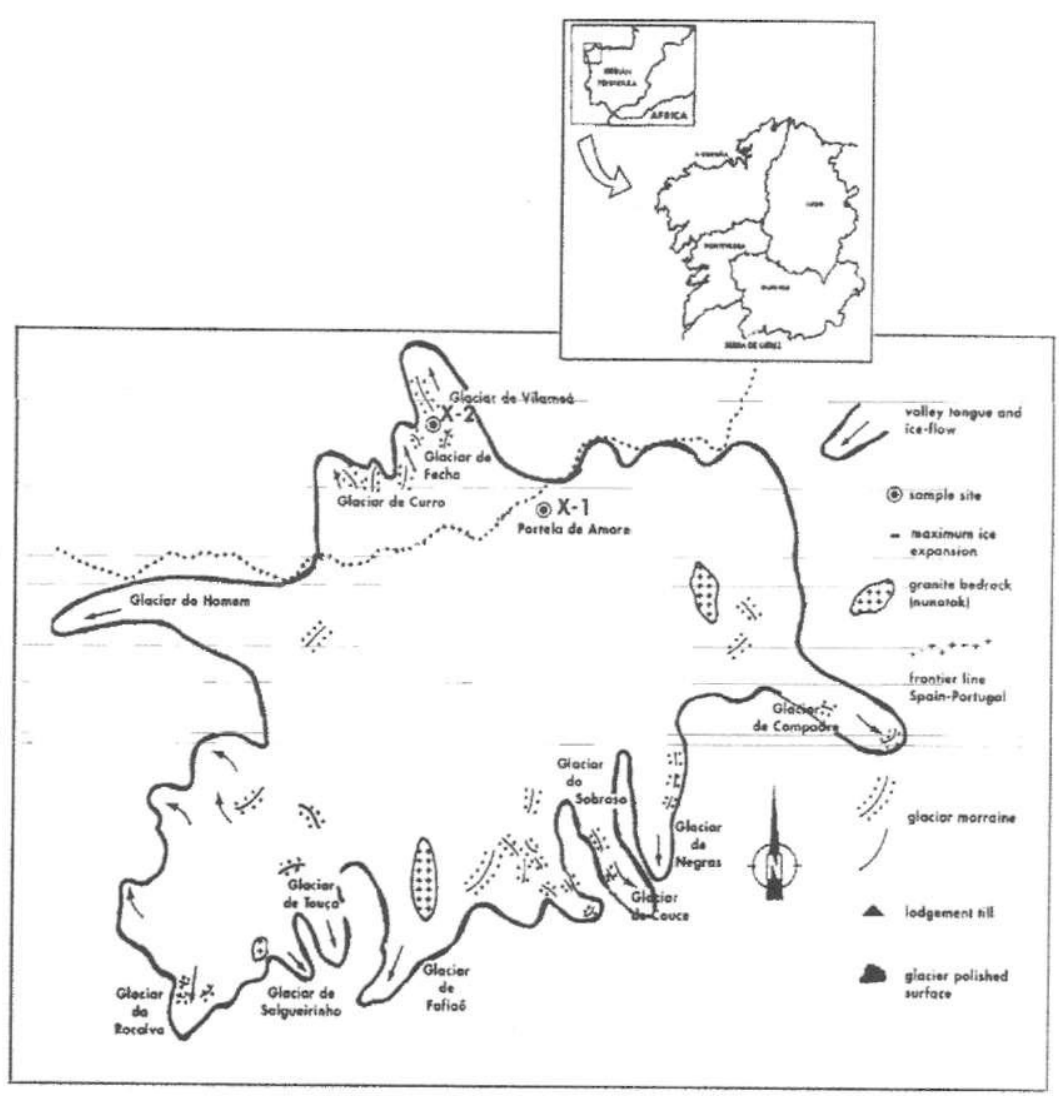

Fig. 1 - Síntesis de la cartografia geomorfológica glaciar de la Serra de Xurés-Gerêz, (Galicia-Portugal, NW ibérico), con la situación de las muestras datadas por isótopos cosmogénicos.
Fig. 1 - Synthesis of glacial geomorphological cartography of Serra de Gerês (Galicia-Portugal, NW Iberia), with the situation of samples dated by cosmogenic ${ }^{21} \mathrm{Ne}$ isotopes. 


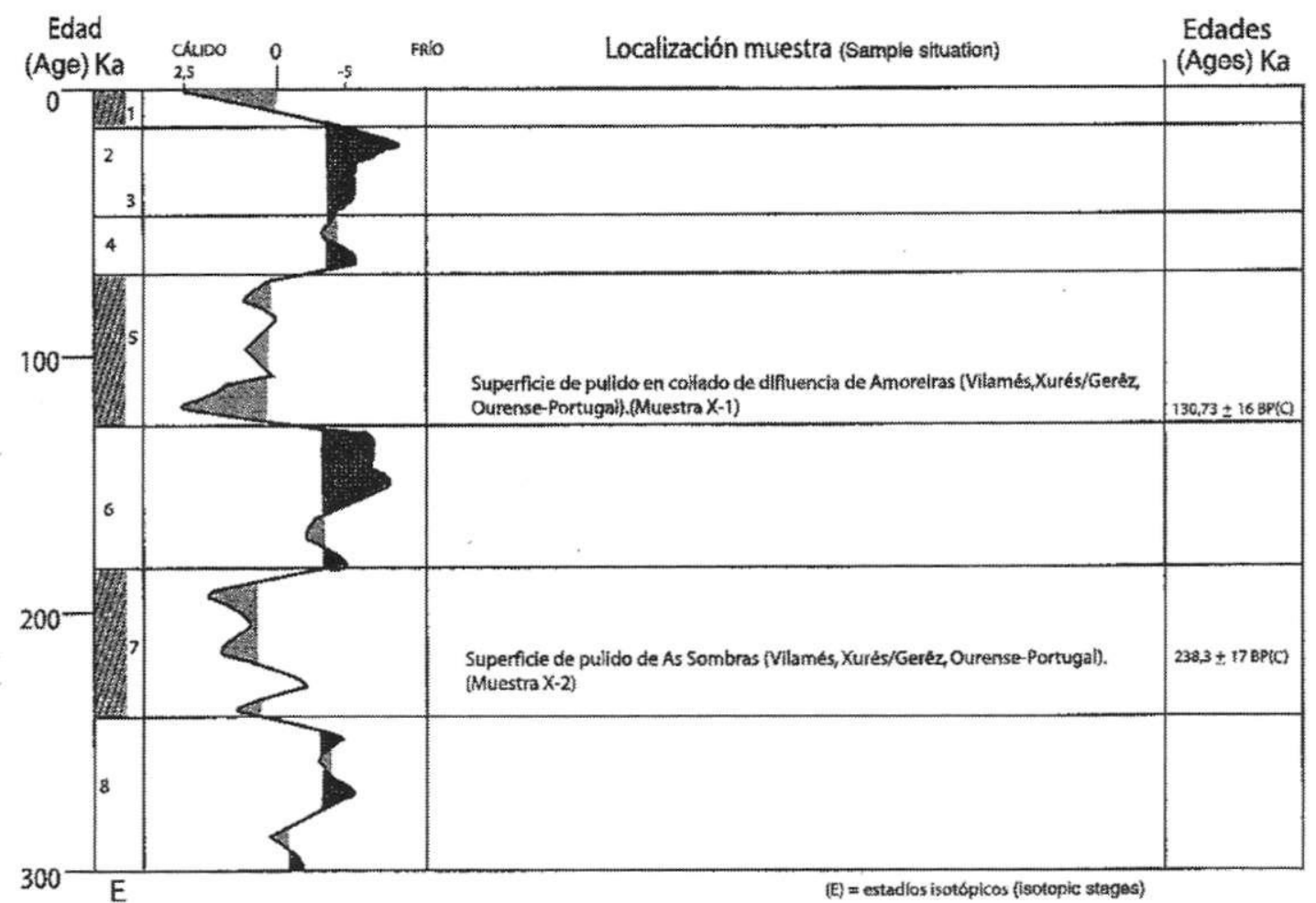

Fig. 2 - Escala de temperaturas para el Cuaternario Superior a partir del registro isotópico de oxígeno en sedimentos oceánicos (BERGer et al., 1984, modificado).
Fig. 2 - Temperature variation for the Upper Quaternary from the isotopic registers in oceanic sediments (modified from BERGER et al., 1984)

\begin{tabular}{|l|l|l|}
\hline Muestra/sample & Coord.Geograficas/Geographical coordenates & Altitud s.n.m./altitude o.p.s.l. \\
\hline $\mathrm{X}-1$ & $41 .^{\circ} 48^{\prime} 932 \mathrm{~N} / 8 .^{\circ} 3{ }^{\prime} 110 \mathrm{~W}$ & $1300 \mathrm{~m}$ \\
$\mathrm{X}-2$ & $41 .^{\circ} 49^{\prime} 700 \mathrm{~N} / 8 .^{\circ} 4^{\prime} 109 \mathrm{~W}$ & $1100 \mathrm{~m}$. \\
\hline
\end{tabular}

Tabla 1 - Coordenadas de las muestras seleccionadas para su datación. Se indica asimismo su altura sobre el nivel del mar.
Table 1 - Geographical coordinates for the location of the studied samples. The altitude over the present sea level is indicated. finalizada esta operación se procedió a la separación y purificación del cuarzo. La fracción elegida fue la comprendida entre $0,5 \mathrm{~mm}<0<0,95 \mathrm{~mm}$.

\subsection{Separación del cuarzo}

Para la separación del cuarzo del resto de minerales que forman la roca se modificó el proceso habitual (KOHL \& NISHIIZUMI, 1992), sometiendo la muestra a una digestión en $\mathrm{HC} 1$ concentrado durante tres horas a $85 .{ }^{\circ} \mathrm{C}$ con el fin de disolver los óxidos de hierro. A continuación se procedió a una digestión en $\mathrm{HF}(1 \%) / \mathrm{HNO}_{3}(1 \%)$ otras tres horas a $55 .{ }^{\circ} \mathrm{C}$ en baño de ultrasonidos. Seguidamente se realizó a una separación por densidades entre la mica y el cuarzo utilizando una disolución de politungstenato sódico de densidad $2,65 \mathrm{~g} / \mathrm{cm}^{3}$ apoyado por ultracentrifugación. En la etapa final se procedió a disolver la parte más externa de los granos de cuarzo para minimizar en lo posible los aportes de $\mathrm{Ne}$ producido en las reacciones ${ }^{18} 0(\mathrm{a}, \mathrm{n})^{21} \mathrm{Ne}$ y ${ }^{\cdot 9} \mathrm{~F}(\mathrm{cc}, \mathrm{n})^{22} \mathrm{Ne}$. Para ello se realizaron dos digestiones en HF 1:8 de 20 minutos de duración en baño de ultrasonidos y se completó el proceso de separación con un examen a la lupa binocular con el fin de eliminar posibles impurezas, (fases minerales diferentes al cuarzo) no eliminadas previamente. 


\subsection{Litólogía}

Serra de Gerés: Es un cuerpo intrusivo (FERNANDEZ TOMÁS \& PILAS MATEO, 1974a y b) granodiorítico biotítico de grano medio a grueso. Según COTTARD (1979), X-1 es del tipo local llamado granito de grano fino de Carrís. La muestra X-2 es de granito porfíroide de Xerés (COTTARD, 1979).

\subsection{Determinación de ${ }^{2} \mathrm{Ne}$ cosmogénico}

Todos las medidas de ${ }^{21} \mathrm{Ne}$ fueron realizadas con la técnica de Espectrometría de Masas de Relaciones Isotópicas de Gases Nobles (NG-IRMS) (FERNANDEZ MOSQUERA, 1999). Aunque el ${ }^{21} \mathrm{Ne}$ tiene parcialmente un origen cósmico también puede ser generado en los procesos petrogenéticos por la inclusión de fluidos/ gases durante la consolidación de la roca (componente heredado) que aportará una composición isotópica relacionada con las condiciones de la cámara magmática. Las reacciones de desintegración nucleares (oc,n), de los minerales de la roca después de su consolidación (componente nucleogénico) aportan también partículas a. Estas tres fuentes: nucleogénica, heredada y cosmogénica contribuyen a la concentración total del $\mathrm{Ne}$ en la roca (NIEDER-MAN et al., 1994). Ocurre entonces que el componente nucleogénico más el heredado pueden contribuir de manera apreciable a la magnitud alcanzada por este isótopo al ser procesos iniciados después de la consolidación de la roca. En los granitos de Xurés-Gerés, esto ocurre hace aproximadamente 300 millones de años (ver D E N T EX, 1978), frente a los menos de 300 ka años de exposición a los rayos cósmicos de las superficies rocosas muestreadas.

\subsection{Caracterización geoquímica}

Las muestras seleccionadas se caracterizaron por su contenido en elementos productores de partículas _que podrían alterar la proporción de la fracción cosmogénica del ${ }^{2} \mathrm{Ne}$. En primer lugar se calculó el tanto por ciento en $\mathrm{SiO}_{2}$ por gravimetría, (cuando está en forma de cuarzo es el principal productor de la fracción cosmogénica de ${ }^{21} \mathrm{Ne}$ ). Se analizaron además (por ICP-MS) el contenido en otros elementos que también contribuyen a la formación de isótopos cosmogónicos fueran estos radioactivos (K, U, Th) o no, (Be, Na, Mg, Al, P, Ca, Mn, Fe) (ver tabla 2). De esta forma se pudo comprobar, la adecuación en todos los casos de todas las muestras seleccionadas para la datación por isótopos cosmogónicos por la relación conté- nido en $\mathrm{SiO}_{2} /$ otros elementos productores de partículas a.

\begin{tabular}{|l|l|l|}
\hline & $\mathrm{X}-1$ & $\mathrm{X}-2$ \\
\hline $\mathrm{SiO}_{2} \%$ & $60,3 \pm 0,2$ & $59,9 \pm 0,5$ \\
\hline${ }^{9} \mathrm{Be} \mu \mathrm{g} / \mathrm{g}$ & $7,6 \pm 0,5$ & $6,8 \pm 0,2$ \\
\hline${ }^{23} \mathrm{Na} \mathrm{mg} / \mathrm{g}$ & $33,4 \pm 2,2$ & $29,6 \pm 0,1$ \\
\hline${ }^{24} \mathrm{Mg} \mathrm{mg} / \mathrm{g}$ & $1,0 \pm 0,07$ & $1,8 \pm 0,03$ \\
\hline${ }^{27} \mathrm{Al} \mathrm{mg} / \mathrm{g}$ & $74,9 \pm 6,3$ & $70,8 \pm 0,7$ \\
\hline${ }^{31} \mathrm{P} \mu \mathrm{g} / \mathrm{g}$ & $<25$ & $<25$ \\
\hline${ }^{39} \mathrm{~K} \mu \mathrm{g} / \mathrm{g}$ & $34,5 \pm 2,9$ & $30,5 \pm 0,1$ \\
\hline${ }^{44} \mathrm{Ca} \mathrm{mg} / \mathrm{g}$ & $3,5 \pm 0,2$ & $8,1 \pm 0,2$ \\
\hline${ }^{55} \mathrm{Mn} \mathrm{mg} / \mathrm{g}$ & $0,29 \pm 0,02$ & $0,28 \pm 0,01$ \\
\hline${ }^{56} \mathrm{Fe} \mathrm{mg/g}$ & $9,4 \pm 0,8$ & $10,2 \pm 0,1$ \\
\hline${ }^{232} \mathrm{Th} \mu \mathrm{g} / \mathrm{g}$ & $24,7 \pm 0,3$ & $17,7 \pm 0,4$ \\
\hline${ }^{238} \mathrm{U} \mu \mathrm{g} / \mathrm{g}$ & $11,5 \pm 0,9$ & $3,9 \pm 0,1$ \\
\hline
\end{tabular}

Tabla 2. - $\mathrm{SiO}_{2}$ medido por gravimetría y análisis elemental por ICP-MS de las muestras seleccionadas.

Table 2. - gravimetry measures of $\mathrm{SiO}_{2}$ and elemental analysis by ICP-MS for studied samples.

\section{Resultados}

\subsection{Valores de edades para las muestras}

A continuación se presenta el cuadro de edades obtenido para las muestras descritas (tabla 3 ).

\begin{tabular}{|l|l|}
\hline $\begin{array}{l}\text { Código de muestra/ } \\
\text { sample code }\end{array}$ & $\begin{array}{l}\text { Edad de Exposición (Kaños)/ } \\
\text { Exposition age in ky. }\end{array}$ \\
\hline X-1 & $130,7 \pm 16,8$ \\
X-2 & $238,3 \pm 17,2$ \\
\hline
\end{tabular}

Tabla 3 - Edades de las muestras estudiadas en Serra do Gêres (ver fig. 1).

Table 3 - Age of the samples from Serra do Gêres (see fig. 1).

\section{DISCUSIÓN}

Las edades obtenidas para las muestras estudiadas son coherentes con el esquema geomorfológico previo al que además añaden mayor precisión. Según nuestra interpretación de los hechos, a partir del último máximo glaciar, la fusión del casquete de hielo dejó al descubierto el macizo rocoso previamente degradado por la erosión glaciar. En consecuencia, las edades de las muestras desde las zonas mas externas 
a las mas internas del área glaciada son gradualmente cada vez mas modernas, (Ver figuras 1 y 2). Dado que la superficie afectada por la glaciación es muy reducida parece lógico suponer que las superficies datadas corresponden a la erosión durante fases glaciares diferentes, (obsérvese, Tabla 3, que hay diferencias de unos $100 \mathrm{ka}$ años entre la muestra X-2 y la X-1). La edad de la muestra X-1, $130 \mathrm{ka}$ años B.P. es sustancialmente mayor de lo supuesto hasta el momento, (las estimaciones para el último máximo glaciar se situaban hasta ahora entre $40 \mathrm{ka}$ y $50 \mathrm{ka}$ B.P., (ver GRANDAL et al, 1997 y VIDAL ROMANI, 1997). Al situarse la muestra en la divisoria de la Pórtela da Amoreira (fig. 1), señala aproximadamente el momento la última difluencia desde la zona portuguesa a la española, marcando por tanto el inicio de la última deglaciación general en toda el área. También aporta un dato muy importante y es que el máximo glaciar de, aproximadamente hace 21 ka, durante el máximo weichseliense, (ANDERSEN \& BORNS, 1994), no fue en esta zona, y tampoco en el resto del Noroeste, el mas importante. Por el contrario la edad obtenida para la otra muestra - X-2 (ver figura 1) de 238.300 años B.P, corresponde a una etapa de máximo avance glaciar, cuando la difluencia alcanzó su mayor entidad (VIDAL ROMANI et al, 1990a y b). La situación de X-2 entre 3 cordones morrénicos: uno de ellos correspondiente a la pulsación mas avanzada señalada hasta el momento, los otros dos a una pulsación posterior, (BRUM FERREIRA et al., 1992) bien que todas dentro de la etapa de máxima difluencia, indica que la edad del avance glaciar que representa el pulido sería aun mas antigua que la señalada por X-2 y por lo tanto entraría de lleno dentro del estadio isotópico 8 (entre 250.000-300.000 años B.P.). Las implicaciones que tiene esta cronología son evidentes ya que permiten la mayor precisión conseguida hasta el momento en la cartografía geomorfológica, (VIDAL ROMANI et al., 1990a y b; BRUM FERREIRA et al., 1992), pues hasta ahora se presumía una edad mas moderna para la deglaciación en el área y se asignaban todos los depósitos a la misma fase glaciar. Con la nueva cronología se distinguen al menos 2 estadios isotópicos (6 y 8), diferentes donde hasta el momento solo se distinguía una prolongada deglaciación, desde el estadio 4 ó 3, (ver BORDONAU I IBERN, 1992; BRUM FERREIRA et al, 1992; GRANDAL et al., 1997; JIMÉNEZ SÁNCHEZ, 1996; LEIRA \& VIDAL ROMANI, 1997; VIDAL ROMANÍ, 1979, 1996; VIDAL ROMANÍ \& SANTOS, 1993, 1994; VIDAL ROMANí et al, 1995). Esta idea se ve reforzada por las dataciones obtenidas en Serra de Queixa-Invernadoiro (VIDAL ROMANI et al, 1999). En este trabajo se presentan los resultados obtenidos con solo 2 muestras datadas por elementos cosmogónicos pero es evidente que la realización de mas dataciones permitirá una mejor caracterización cronológica y dinámica de la glaciación.

\section{CONCLUSIONES}

El estudio de la dinámica glaciar en zonas como el NW Peninsular, donde el glaciarismo se desarrolló sobre sustrato granítico, pobre en sedimentos y estos poco diferenciados, lo que no sucede en áreas de rocas sedimentarias, había planteado hasta ahora un gran número de dificultades. Basado exclusivamente en datos geomorfológicos no se había podido establecer una secuencia suficientemente detallada, y mas aun cuando como en Gerés el glaciarismo se desarrolló reiteradamente durante el Pleistoceno sobre una misma y reducida superficie. Por otra parte los registros sedimentarios de áreas graníticas con un componente detrítico predominante han hecho imposible la datación de los sedimentos glaciares por métodos absolutos. Finalmente, esta el carácter particular de estos glaciares desarrollados en macizos montañosos aislados, sin posibilidad alguna de conectarse a otros mejor conocidos o estudiados, con áreas de drenaje obviamente locales y con secuencias de terrazas imposibles de correlacionar con la red de drenaje regional o con incluso niveles marinos. Todo ello hace especialmente relevante el método de datación como los isótopos cosmogónicos, que permiten fijar la edad de exposición a la radiación cósmica de una superficie rocosa glaciada. Los datos obtenidos se adaptan al modelo de evolución del clima durante la última etapa del Cuaternario y deducido a partir del sondeo de hielo de Vostok. (PETIT et al, 1999). Según esto, en las dos áreas estudiadas y a tenor de las muestras analizadas hasta el momento, estaría reflejada la evolución de los sistemas glaciares aproximadamente durante los últimos $300 \mathrm{ka}$.

\section{A GR A DECIMIENTOS}

Los autores de este trabajo desean agradecer a la Dra. Luisa Santos Fidalgo su síntesis bibliográfica de datos palinológicos de la Serra de Gerés.

\section{B IB LIOGR AFÍA}

ANDERSEN, B. G. BORNS, H.W. (1994) - The ice age world. Scandinavian University Press, Oslo, 208p.

BELLOT RODRÍGUEZ, F. (1952) - El análisis polínico de las zonas higroturbosas de la Sierra de Geres en relación con las presencia de Pinus pinaster Sol. in ait. y Pinus sylvestris L, Agronomia Lusitana, 12 (3), p. 481-491.

BORDONAU I IBERN. (1992) - Els complexos glàcio-lacustres relacionais amb el darrer cicle glacial als Pirineus, Tesis Doctoral, Universität de Barcelona, 294 p. 
BRUM FERREIRA, A.; VIDAL ROMANI, J. R.; VILAPLANA, J. M. RODRIGUES, M. L.; ZÊZERE, J. L. \& MONGE, C. (1992) Formas e depósitos glaciarios e periglaciários da Serra do Gerêz-Xurés (Portugal; Galicia). Levantamento cartográfico, Cuadernos do Laboratorio Xeolóxico de Laxe, 17, p. 121-135.

BRUM FERREIRA, A.; VIDAL ROMANÍ, J. R.; ZÊZERE, J. L \& RODRIGUES, M. L. (1999). A Glaciação Plistocenica da Serra de Gerês. Vestigios geomorfológicos e sedimentológicos, C.E.G, Área de Geografía Física e Ambiente, Relatório 37, Lisboa, $150 \mathrm{p}$.

CERLING, T. E. \& CRAIG, H (1994) - Geomorphology and in situ cosmogenic isotopes, Annual Review of Earth and Planetary Science, 22, p. 273-317.

CHOFFAT, P. (1894) - Promenade au Gérez. Souvenirs d'un géologue, Lisboa, $18 \mathrm{p}$.

COTTARD, F. (1979) - Petrologie structurale et métallogénie du complexe granitique de Lovios-Gerêz. Le modèle de mise en place de la mine de Las Sombras (Sn-W-Mo-Bi) (Sud GaliceEspagne), Tesis Doctoral, Paris, $226 \mathrm{p}$.

COUDÉ-GAUSSEN, G. (1981) - Les Serras da Peneda et do Gerês. Étude Géomorphologique, Memorias do Centro de Estudos Geográficos, 5, Lisboa, 254 p.

COUDE-GAUSSEN, G. (1978) - Confirmation de l'existence d'une glaciation wtirmienne dans les montagnes du NW du Portugal, Comptes Rendues sommaires de la Société Géologique de France, 1, p. 34-37.

COUDE-GAUSSEN, G. (1980) - Les serras orientales du Minho (Portugal): orogenèse, modelé granitique et altérations, Revue de Géographie des Pyrénées et du Sud-Ouest, 51-3, p. 291-313.

COUDÉ-GAUSSEN, G. \& DENÈFLE, M. (1980) — La signification du développement récent de la lande d'altitude dans le Portugal Septentrional d'après l'étude de deux tourbières. Bulletin de l'A.F.E.Q., 3, p. 107-115.

DEN TEX, E. (1978) — El zócalo policíclico y su importancia en la evolución de la Cadena Varísca en Galicia Occidental, in ANTHONIOZ, BALDWFN, BARD, BUISKOOL, CAPDEVILA, CORRETGÉ, DEN TEX, FERRAGNE, HAALEBOS, JULIVERT, MARTÍNEZ, MATTE, MINNIGH, OVERMEEREN, SCHMIDT -THOMÉ, TEIXEIRA, VEGAS Y ZAMARREÑO (eds.), Geología de la parte Norte del Macizo Ibérico. Edición Homenaje a Isidro Parga Ponda, Cuadernos do Seminario de Estudos Cerámicos de Sargadelos, 27. O Castro, Sada, A Corana, España, p. 139-157.

FERNÁNDEZ TOMÁS \& PILAS MATEO, E. (1974a) — Mapa Geológico de Lovios. Hoja 301. Serie MAGNA. Servicio de Publicaciones I.G.M.E. Madrid.

FERNÁNDEZ TOMÁS \& PILAS MATEO E. (1974b) — Mapa Geológico de Portela d'Home. Hoja 336. Serie MAGNA. Servicio de Publicaciones I.G.M.E. Madrid.

FERNÁNDEZ MOSQUERA, D. (1999) —Aplicación de los nucleidos cosmogónicos a la datación de procesos geológicos, Tesis de Licenciatura, Instituto Universitario de Geología Isidro Parga Pondal, Universidade de A Corana, A Corana, 114 p.

GRANDAL, A.; LOPEZ GONZALEZ, F. \& VIDAL ROMANI J. R. (1997) Condicionantes en la distribución de macromamiferos en Galicia (NW Península Ibérica) durante el Cuaternario superior. Cadernos do Laboratorio Xeolóxico de Laxe, 22, p. 43-66.

GUTIÉRREZ, G (1957) — Notas sobre la Sierra de Jurés (Ourense). Notas y Comunicaciones del Instituto Geológico y Minero de España. Inst. Geol. Min. Esp., 45, p. 27-36.

JIMÉNEZ SÁNCHEZ, M. (1996) - El glaciarismo en la cuenca alta del Rio Nalón (NO de España): una propuesta de evolución de los sistemas glaciares cuaternarios en la Cordillera Cantábrica. Revista de la Sociedad Geológica de España, Madrid, 9 (3-4), p. 157-168.

KOHL, C. P. \& NISHIIZUMI, K. (1992) - Chemical isolation of quartz for measurement of in situ produced cosmogenic isotopes, Geochimica et Cosmoquimica Acta, 56, p. 3583-3587.
LEIRA CAMPOS, M. F. \& VIDAL ROMANI, J. R. (1997) - Evolución postglaciar de la laguna de Villaseca (NW de la Península Ibérica), a partir del análisis de diatomeas. Cadernos do Laboratorio Xeolóxico de Laxe, 22, p. 81-98.

MARTíNEZ DE PISÓN, E. \& ARENILLAS PARRA, M. (1979) — Algunos problemas de morfología glaciar en la España atlántica, Acta Geológica Hispánica, 14, p. 445-450.

NIEDERMANN, S.; GRAFT, T.; KIM J. S., KOHL C. P; MARTI, K. \& NISHIIZUMI, K. (1994) - Cosmic-ray-produced $21 \mathrm{Ne}$ in terrestrial quartz: the neón inventory of Sierra Nevada quartz separates, Earth and Planetary Science Letters, 125, p. 341-355.

MARTÍNEZ DE PISÓN, E.; ARENILLAS PARRA, M. (1984) — Nuevos problemas de morfología glaciar en la España Atlántica, Estudios Geográficos, 175, p. 159-174.

PETIT, J. R.; JOUZEL, J.; RAYNAUD, D.; BARKOV, N. L; BARNOLA, J. M.; BASILE, I.; BENDER, M.; CHAPELLAZ, J.; DAVIS, M.; DEAYGUE, G; DELMOTTE, M.; KOTLYAKOV, V. M.; LEGRAND, M.; LIPENKOV, V.Y.; LORIUS, C; PEPIN, L.; RITZ, C; SALTZMAN E. \& STIEVENARD, M. (1999) - Climate and atmospheric history of the past 420,000 years from the Vostok ice core, Antárctica, Nature, 399, p.429-436.

RAMIL REGO, R; AIRA RODRÍGUEZ, M. J. \& MATÍAS ALONSO, F. (1993) - Caracterización climática y vegetacional de la Serra do Gerês (Portugal) durante el Tardiglaciar y el Holoceno: análisis polínico de Lagoa do Marinho. Actas da 3. ${ }^{\circ}$ Reunião do Quaternário Ibérico, Coimbra, p. 85-92.

RAMIL REGO, F.; TABOADA CASTRO, T. \& AIRA RODRÍGUEZ, M. J. (1996) - Modificación de la cubierta vegetal y acción antrópica en la región del Minho (Norte de Portugal) durante el Holoceno. Biogeografía Pleistocena-Holocena de la Península Ibérica, p.199-214.

RAMIL REGO, P.; MUÑOZ SOBRINO, C; RODRIGUEZ GUITIAN, M. \& GOMEZ ORELLANA, L. (1998) - Differences in the Vegetation of the North Iberian Penninsula during the last 16.000 years. PlantEcology, 138, p. 41-62.

Ruiz ZAPATA, B.; CORREIA, A. I.; DAVEAU, S. \& LECOMPTE, M. (1993) - Datos preliminares sobre la evolución de la vegetación en las sierras del Noroeste de Portugal, Actas da 3." Reunião do Quaternário Ibérico, Coimbra, p. 97-104.

SCHMIDT-THOME, P. (1973) — Neue niedrig gelegene zeugen einer wurmeis-zetlichen vergletscherung im norateil der Iberischen halbinsel, Eizeitalter und Gegenwart, 24, p. 384-389.

SCHMIDT-THOMÉ, P. (1978) - Nuevos testigos de una glaciación wurmiense extensa y de altura muy baja en el NW de la Península Ibérica. Ourense-Pottugal. Geología de la parte $\mathrm{N}$ del MHP, Cuadernos del Seminario de Estudios Cerámicos Sargadelos, 27, p. 221-243

SCHMIDT-THOMÉ, P. (1983) - Besonders niedrig gelegene zeugen einer wurmeiszeitlichen vereibung in nord-west Spanien und nord Portugal, Spat-imd Postglaziale Gletscherschwankungen Glazial und Periglazial Formen. Colloquium, 1980, Rotterdam, p. 213-230.

SOARES DE CARVALHO, G. \& LOPES NUNES, J. (1981a) - A problemática dos indicios glaciarios quaternários na Serra do Gerez e na Serra da Peneda (Portugal), Cuadernos do Laboratorio Xeolóxico de Laxe, 2, p. 289-296.

SOARES, G. \& LOPES NUNES, J. E. (1981b) - Comentarios sobre imagens de factos denunciantes de processos glaciarios quaternários nas Serras do Gerés e da Peneda Portugal, Memorias e Noticias, Publ. Mus. Lab. Mineral. Geol. Univ. Coimbra, 91-92, p. $164-171$

TEIXEIRA, C. \& CARDOSO, J. L. (1979) — A nao confirmação de fenómenos glaciarios nas montanhas do Norte de Portugal (Peneda-Geres), Boletim da Sociedade Geológica de Portugal, XXI fasc.II-III, p. 163-184.

VIDAL ROMANI, J. R.; BRUM FERREIRA, A.; ZÊZERE, J.; RODRIGUES, L. \& MONGE, C. (1990a) - Evolución cuaternaria del relieve granítico en la Serra de Gerês-Xurés (Minho Portugal y Ourense Galicia), Cuaternario y Geomorfología , 4, p. 3-12. 
VIDAL ROMANÍ, J. R.; VILAPLANA, J. M.; BRUM FERREIRA, A.; ZEZERE, J.; RODRIGUES, M. L. \& MONGE, C. (1990 b) Estudio de los till subglaciares de la Serra de Geréz-Xurés (Minho Portugal Ourense Galicia), Cuaternario y Geomorfología, 4, p. 13-25.

VIDAL ROMANÍ, J. R.; FERNANDEZ MOSQUERA, D.; MARTI, K. \& BRUM FERREIRA, A. (1999) - Nuevos datos para la cronología glaciar pleistocena en el NW de la Península Ibérica, Cadernos do Laboratorio Xeolóxico de Laxe, 24, O Castro, Coruña, España, p. 7-29.

VILAPLANA, J. M. (1981) — El método de trabajo utilizado en el estudio del glaciarismo cuaternario del Pirineo y su posible aplicación en las sierras galaico-portuguesas, Cuadernos do Laboratorio Xeolóxico de Laxe, 2, O Castro, España, p. 259-288. 